\title{
Short-term prediction of freeway travel times by fusing input-output vehicle counts and GPS tracking data.
}

\author{
Margarita Martínez-Díaz ${ }^{a^{*}}$ and Francesc Soriguera ${ }^{\mathrm{b}}$ \\ ${ }^{a}$ Assistant Professor, A Coruña Civil Engineering School, Universidade da Coruña, A Coruña, Spain \\ ${ }^{\mathrm{b}}$ Associate professor, BIT-Barcelona Innovative Transportation. Barcelona Civil Engineering School. UPC-BarcelonaTech, Barcelona, Spain
}

\section{ABSTRACT}

Short-term travel time prediction on freeways is the most valuable information for drivers when selecting their routes and departure times. Furthermore, this information is also essential at traffic management centres in order to monitor the network performance and anticipate the activation of traffic management strategies. The importance of reliable short-term travel time predictions will even increase with the advent of autonomous vehicles, when vehicle routing will strongly rely on this information. In this context, it is important to develop a real-time method to accurately predict travel times. The present paper uses vehicle accumulation, obtained from input-output diagrams constructed from loop detector data, to predict travel times on freeway sections. Loop detector count drift, which typically invalidates vehicle accumulation measurements, is corrected by means of a data fusion algorithm using GPS measurements. The goodness of the methodology has been proven under different boundary conditions using simulated data.
\end{abstract}

\section{Introduction and background}

Travel time is, and will continue being, key information for both, traffic management centers and drivers. When received in real time, it allows quantifying the level of congestion on a road link and represents a valuable input for the activation and calibration of dynamic traffic management systems. In turn, travel time information allows drivers to adapt departure times or to change their routes, if possible. In any case, information of expected delays reduces drivers' stress and allows planning later activities accordingly (van Hinsbergen et al., 2007; Soriguera, 2014; Mori et al., 2015; Martínez-Díaz, 2018). In the next future, the value of travel time information will even increase with the popularization of highly automated vehicles, with more technology deployed in the infrastructures, and with new transportation schemes, like Mobility as a Service (MaaS), requiring better information and a higher degree of coordination between transportation modes.

Today, many freeway travel time information systems rely on direct measurements of travel time (e.g. industry solutions such as TomTom, HERE, Google, etc.), although the automatized direct measurement of travel times at traffic management centers is relatively recent. Automatic Vehicle Identification (AVI) or tracking technologies are generally used as measurement devices. AVI identifies individual vehicles at consecutive locations on the freeway and by comparing the timestamps, their travel times are obtained (Turner et al, 1998; Buisson, 2006; Coifman and Krishnamurthya, 2007; Barceló and Kuwahara, 2010; Soriguera et al. 2010; Abott-jard et al., 2013; Bhaskar and Chung, 2013). AVI technology may rely on license plate recognition cameras (LPR), Bluetooth signal detectors or toll tag identification systems, among others. Information is obtained at the individual vehicle level, which may be useful (e.g. for the construction of $\mathrm{O} / \mathrm{D}$ matrices), although travel time information systems rely on the average value computed over a preestablished time interval, $\Delta T$. Travel time information systems using AVI devices suffer from two main drawbacks. First, travel times are only obtained between control points where AVI devices are located, which may be kilometers apart. Second, directly measured travel times provide information form the past, which may be obsolete when feeding real-time information systems. Note that vehicles entering a freeway section want information about their expected travel time, while they would receive information about the average travel time of the vehicles that have exited the section during the last $\Delta T$, at best. This is why the AVI type of travel time information is named arrivalbased travel times (ATT). These can be significantly different than predicted travel times (PTT), especially in changing traffic conditions, if distance between AVI devices is large or if $\Delta T$ lasts long (Soriguera and Robusté, 2011). The alternative for the direct measurement of travel times consists in tracking the vehicles by using GPS or mobile geolocation (e.g. Bar-Gera, 2007; Herrera et al, 2010; Unde and Borkar, 2014), although mobile geolocation is more inaccurate, and given the prevalence of GPS equipped smartphones, a kind of an old fashion need. In this case, travel times are not captive of control points, and can be measured between any two locations on the road. In addition, the obtained information includes the travel times of vehicles on the target section until the present instant, even of those which have only covered the section partially. Therefore, these measurements, called instantaneous travel times (ITT), represent the last information available from vehicles' trajectories. While, form a real-time information perspective, ITT are more desirable than ATT, they are still different than PTT.

Despite the year-to-year increase in the usage of direct travel time measurements, most traffic agencies rely on indirect estimations of travel time, yet. This is due to the fact that inductive-loop detectors are still the most widespread surveillance technology in freeways. In such context, travel times are indirectly estimated from loop detectors' measurements, commonly using the so-called spot-speed methods. These consist in extrapolating the average speed measured at the loops over extended freeway lengths. Spot-speed methods yield ITT estimations, although their accuracy is questionable (Soriguera and Robuste, 2011) since spatial speed extrapolations are mathematical exercises, without considering traffic dynamics and queue evolution.

In conclusion, most of the travel time estimation methods currently used for real-time information either yield ATT or ITT, avoiding the complexities and uncertainties of predictions (PTT). This does not mean that there are not research works in the literature dealing with short-term freeway travel time prediction. On the contrary, literature is vast. Conceptually, different types of approaches have been proposed, which can be classified in three groups (van Lint et al. 2005): i) Model-based approaches, where traffic flow models are used to predict traffic evolution, and from here, travel-time predictions are derived. Examples can be found using DynaMIT (Ben-Akiva et al., 2002), DynaSMART (Hu, 2001) or METANET (Smulders et al., 1999). ii) "Instantaneous" travel time predictors, which use current and past traffic flow variables, such as speeds or flows, to predict travel times, assuming that traffic conditions will remain stationary for an indefinite time period (see for example (Van Lint and Van der Zijpp, 2003)). And iii) data-driven approaches, 
either using parametric methods (e.g. linear regression (Zhang and Rice, 2003; Sun et al., 2003; Laoide-Kemp and O'Mahony, 2020), time series models (Yang, 2005; Min and Wynter, 2011; Ishak and Al-Deek, 2002), Kalman filtering (Okutani and Stephanedes, 1984; Chien and Kuchipudi, 2003; Nanthawichit et al., 2003; Chu et al., 2005; Van Lint, 2008; Xia et al., 2011)) or non-parametric methods (e.g. support vector regression (Lam and Toan, 2008) and various neural network models (Park and Rilett, 1999; Rilett and Park, 2001; van Lint et al., 2002; van Lint et al., 2005; van Lint, 2006; Wei and Lee, 2007; van Hinsbergen et al., 2009; Zeng and Zhang, 2013)).

In spite of this broad amount of works and methods, their usage is limited to off-board navigation solutions in many different variations, but which, from the traffic agencies point of view and as a real-time solution, appear as complex and shady, mostly behaving as black boxes. This happens despite many recent works relying on artificial intelligence and machine learning are relatively easy to implement and provide robust results (see (Zhang and Haghani, 2015) as an example). Besides, more traditional instantaneous methods, based on the measurement of vehicle accumulation (Daganzo, 1983), which do have predictive capabilities as they aim to predict the time required to serve all vehicles accumulated on a freeway stretch, are neither used. This happens despite they could be simply applied using loop detector measurements to construct inputoutput cumulative count curves. Not being an intuitive method, the requirement of vehicle conservation between count measurements, and the loop detector count drift, are amongst the reasons of their lack of popularity. In fact, detector drift is a severe drawback, as vehicle accumulation is obtained from the difference of cumulative counts at consecutive detectors (i.e. input - output). In this context, even a small systematic error in detector counts, when accumulated in time, can make the estimation of vehicle accumulation totally unreliable.

In view of the previous considerations, it would be desirable to take advantage of the predictive capabilities of vehicle accumulation for travel time prediction by developing a simple method for correcting the drift error in input - output cumulative curves. In order to be applied in practice, the method should rely on data commonly available at traffic management centers. Today, data from tracking systems, especially from GPS onboard equipment (e.g. smartphones, tablets, smart watches, cameras, etc. or dedicated GPS trackers) fulfills this criterion. Whereas specific GPS trackers are mainly installed in commercial fleets and public transport (e.g. buses, taxis, etc.) (Leduc, 2008), undedicated GPS signal receptors are widespread, especially in the form of smartphones. Any of these tracking devices allows obtaining GPS data very frequently (e.g. every second) yielding huge databases of raw vehicles' trajectories. Complex applications of these data are possible, such as trajectory reconstruction (Patire et al. 2015; Knapen et al., 2018), estimation of origin-destination (OD) matrices (Ge and Fukuda, 2016; Zhu and Ye, 2018), driving behavior analysis (Vlahogianni and Barmpounakis, 2017; Chen et al., 2019) or travel time estimation (Cedillo-Campos et al, 2019; Krause and Zhang, 2019). In addition, the use of tracking data already plays an important role in the context of the connected driving environment (Ge et. al., 2018; Wang et al., 2020). However, the costs linked to communications, data storage and computational capabilities, are high. These costs are significantly reduced when using low-frequency GPS data (e.g. one sample per minute). In this case, the most typical applications are vehicle positioning, tracking, and remote management of vehicle fleets (Laha and Putatunda, 2018; Kemajou et al., 2019). Recently, neural network architectures fed with low-frequency GPS data, have also shown their potential to provide efficient vehicle classification, considering small-duty, medium-duty and heavyduty vehicles (Simoncini et al., 2018).

In this context, the goal of the present paper is to develop a reliable and generally applicable method for the real-time prediction of travel times based on vehicle accumulation on a freeway section. The main contribution of the paper is the development of a data fusion algorithm using GPS data to correct the drift in input-output diagrams from loop detectors. Specifically, the method consists in estimating ITT from cumulative counts at loop detectors. These ITTs, which are affected by detector drift, are compared with direct ITT from GPS data measurements. By assuming the ITT from GPS tracking data as highly accurate, the detector drift is corrected. This allows constructing corrected input-output diagrams, which are then used to obtain a reliable estimation of vehicle accumulation from where to obtain short-term travel time predictions (PTT). The goodness of the proposed method is proved, even in a simulated hostile context, with severe congestion and only a low percentage of tracked vehicles. Among other issues, the impact on PTT accuracy of the frequency of GPS data collection, as well as the length of the time intervals of aggregation are analyzed in the paper.

The remainder of the paper explains the data fusion methodology in detail, and proves its goodness in a simulated environment. Next, the obtained results as well as the influence of the most important parameters are discussed. The paper ends with a summary of conclusions and the proposal of key issues for further research.

\section{Estimating delays from input - output diagrams}

Input - output cumulative vehicle count diagrams are constructed by plotting the cumulative vehicle count, $N$, versus time, $t$, for two consecutive loop detectors without in between in/out flows. The two detectors define a closed freeway section, $i$, where the cumulative count at the upstream detector constitutes the "arrivals curve", $A_{i}(t)$, and that of the downstream detector the "departures curve", $D_{i}(t)$. Provided that conservation holds in the section and accepting a first in - first out (FIFO) behavior, the horizontal distance between the curves at the height of a vehicle $k$ represents the travel time of this vehicle in the section, whereas the vertical distance between the curves at any time $t$ is the vehicle accumulation in the section at this instant. If $A_{i}(t)$ is translated forward in time a magnitude equal to the free flow travel time in the section, $t_{f(i)}$, a third curve is obtained, called $V_{i}(t)$, the "virtual arrivals curve". $V_{i}(t)$ is simply a translation of $A_{i}(t)$ in time which allows easily determining delays as the horizontal distance between $V_{i}(t)$ and $D_{i}(t)$ in the input-output diagram. Recall that the delay is defined as the difference between the travel time and the free-flow travel time in section $i$. The vertical distance between $V_{i}(t)$ and $D_{i}(t)$ represents the excess accumulation in the section at $t$, defined as $Q_{i}(t)$.

However, some passing usually exists, and the FIFO assumption does not strictly hold. This means that the individual travel times (or delays) obtained with this method would be biased in practice. This does not represent a severe limitation as we are interested in average delays calculated over predetermined time intervals, $\Delta T$. The average ITT delay is estimated from the total cumulative delay drivers spend on section $i$ during the time interval $\Delta T$, which is obtained by computing the enclosed area, $\mathcal{S}_{i}(t)$, between $V_{i}(t)$ and $D_{i}(t)$ during $\Delta T$ (see Fig. 1). Recall that this includes vehicles which only have covered section $i$ partially, and it is not affected by overtakings. So, the average ITT delay per unit distance travelled can be obtained by dividing $\mathcal{S}_{i}(t)$ by the total cumulative distance vehicles have covered on section $i$ during $\Delta T$. This cumulative distance can be estimated as the length of section $i$, defined as $\Delta x_{i}$, times the number of exiting vehicles during $\Delta T$ (i.e. $\left.\Delta x_{i} \cdot\left[D_{i}(t)-D_{i}(t-\Delta T)\right]\right)$; see (Daganzo, 2007) for a more detailed derivation. Finally, $w_{i}(t)$, representing the average ITT delay on section $i$ during $\Delta T$, is obtained by multiplying the delay per unit distance times $\Delta x_{i} . \Delta x_{i}$ cancels out, yielding the result on Equation 1. 


$$
w_{i}(t)=\frac{s_{i}(t)}{D_{i}(t)-D_{i}(t-\Delta T)}
$$

Note that this estimation of $w_{i}(t)$ can be updated every $\Delta t$, where $\Delta t$ is the time interval between loop detector measurements. This has nothing to do with $\Delta T$, the travel time averaging period, although, in practice, the calculations are easier if $\Delta T$ is an integer multiple of $\Delta t$ (i.e. like in Fig. 1 where $\Delta T=2 \Delta t)$.

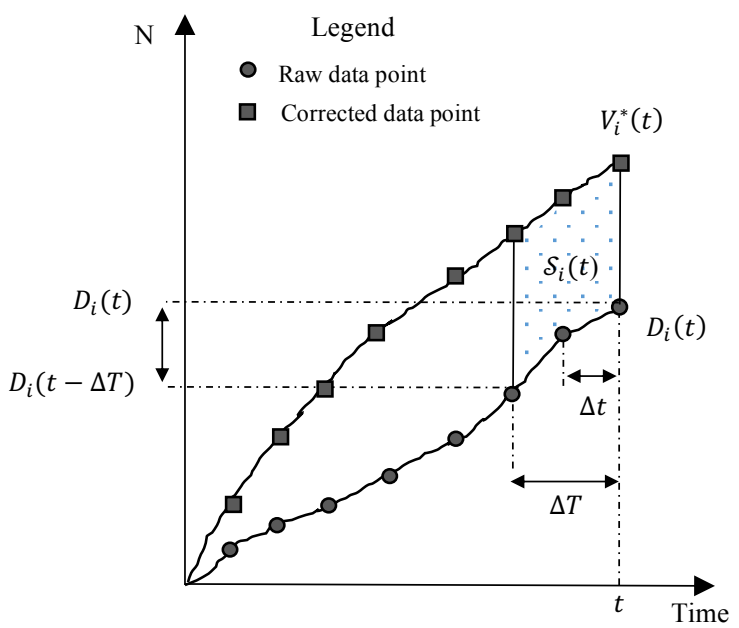

Fig. 1. Estimation of cumulative ITT delay, $\mathcal{S}_{i}(t)$, from input-output diagrams at section $i$ during $\Delta T$. Note: the drift correction procedure yielding $V_{i}^{*}(t)$ (where the subscript " " stands for "corrected") is presented in next sections.

Also note that the estimation of ITT delays in Equation 1, would yield results equivalent to those obtained by averaging all the individual delays obtained by GPS tracking during $\Delta T$. (Soriguera and Martínez-Díaz, 2020) provide further details on how to obtain travel times from input - output diagrams.

From input - output diagrams it is also possible to obtain an estimation of the short-term predicted delay in section $i$ at time $t, w_{i}^{(p)}(t)$, where the subscript ${ }^{(p)}$ stands for "predicted" for all the variables in the paper. $w_{i}^{(p)}(t)$ is obtained by dividing the excess accumulation in section $i$ at time $t, Q_{i}(t)$, by the predicted average outflow from section $i, \bar{q}_{\text {out } \_i}^{(p)}(t)$ (see Fig. 2 and Eq. 2).

$$
w_{i}^{(p)}(t)=\frac{Q_{i}(t)}{\bar{q}_{\text {out_i }}^{(p)}(t)}
$$

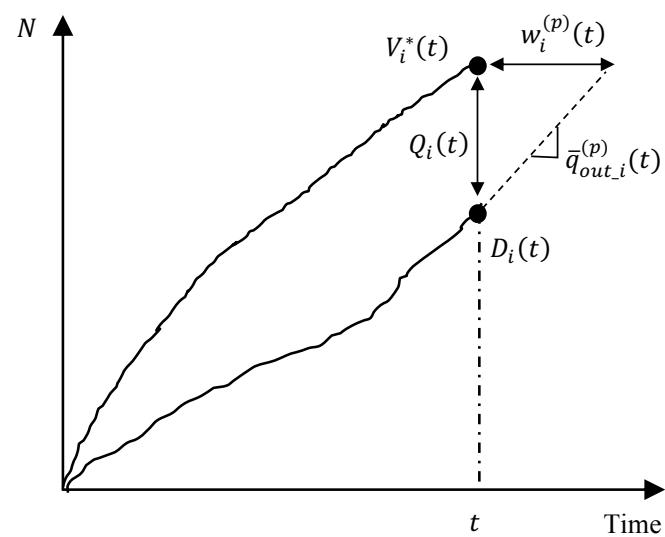

Fig. 2. Estimation of the predicted delay from input-output diagrams.

This estimation of the short-term predicted delay takes advantage of the knowledge of current excess accumulation, $Q_{i}(t)$, in order to predict the time needed to "serve" all vehicles, limiting the forecasting uncertainty to setting the predicted outflow. To that end, $\bar{q}_{\text {out_i }}^{(p)}(t)$ can be set as a moving average of the last measured outflows. The duration, $\tau$, of the time window considered in this moving average affects the performance of the prediction. For example, long averaging periods (i.e. large $\tau$ ) imply a more robust estimation of $\bar{q}_{\text {out } i}^{(p)}$, because of using a larger sample. This will reduce the statistical fluctuations in the estimation of $\bar{q}_{\text {out } \_}^{(p)}$, but it will take longer to incorporate sudden changes in the outflow into the travel time prediction. Short averaging periods (i.e. small $\tau$ ) result in the opposite behavior, favoring immediacy in reporting outflow changes with respect to limiting the fluctuations of predicted travel times. Given this trade-off, it is necessary to find a balance between both factors by calibrating $\tau$ to maximize the accuracy of predictions in any specific application of the method. Just as a reference, the best results were obtained for $\tau=15$ minutes in the application presented later in this paper.

From the expected delay in section $i$ and at time $t, w_{i}^{(p)}(t)$, the expected travel time, $t t_{i}^{(p)}(t)$, is simply obtained by adding the free-flow travel time in the section, defined as $t t_{f_{-} i}$ (see Eq.3).

$$
t t_{i}^{(p)}(t)=t t_{f_{-} i}+w_{i}^{(p)}(t)
$$

Finally, the addition of predicted travel times for consecutive sections allows obtaining corridor travel time predictions.

\section{Using GPS tracking data to correct count drift in input - output diagrams}

The main problem when estimating delays from input - output diagrams constructed from loop detectors is that vehicle count measurements suffer from drift. This is that loop detectors tend to systematically lose a small fraction of passing vehicles. Even though long-term drift correction factors are typically applied to detector counts (i.e. enforcing that over extended periods of time, $24 \mathrm{~h}$ for example, detector counts at consecutive detectors in closed sections coincide), this is not enough to ensure reliable estimation of vehicle accumulation over shorter periods of time. Note that even a small count drift between detectors can turn to be dramatic when accumulated in time to construct $V_{i}(t)$ and $D_{i}(t)$, so that the estimations of $\mathcal{S}_{i}(t)$ and $Q_{i}(t)$ can be totally biased leading to wrong delay estimations.

In this context, in order to use input - output diagrams to estimate travel times and delays, it is necessary to apply a shortterm drift correction method. The proposed method consists in a data fusion algorithm using a moderate amount of direct travel time measurements obtained from GPS tracking data to correct input - output diagrams.

The data fusion algorithm is simple. Every $\Delta T$ (which is generally longer than the loop detector measurement interval, $\Delta t$ ), a directly measured ITT, $\hat{t t}_{i}(t)$, is obtained from GPS tracking data. Note that the "hat" stands for directly measured variables from GPS data. In order to compute $\widehat{t t}_{i}(t)$, consider that each GPS measurement provides, at least, the vehicles' position and timestamp. Then, GPS measurements are filtered to only consider vehicles with two or more data points on section $i$ and time interval $(t-\Delta T, t)$. Using vehicles' position and timestamp this filtering is straightforward. Next, the travel time of vehicle $j$ on the section $i, \widehat{t t}_{i j}(t)$, is obtained as the difference in timestamps between the last and first GPS measurements in the filtered database. The distance covered, $\hat{d}_{i j}(t)$, is obtained analogously from the difference in positions. From these measurements, $\widehat{t t}_{i}(t)$, is obtained as:

$$
\widehat{t t}_{i}(t)=\Delta x_{i} \frac{\sum_{\forall j} \hat{t}_{i j}(t)}{\sum_{\forall j} \hat{d}_{i j}(t)}
$$

Note that the quotient in Equation 4 is the inverse of the average generalized speed (i.e. the average generalized pace) on 
section $i$ and time interval $(t-\Delta T, t)$, as defined by (Edie, 1965). Also note that $\Delta T$ contributes to a more robust estimation of $\widehat{t t}_{i}(t)$, due to a larger sample size, at the price of a less frequent update of the drift correction.

From $\widehat{t t}_{i}(t)$, the directly measured delay, $\widehat{w}_{i}(t)$, is obtained by subtracting the free-flow travel in the section, $t t_{f_{-} i}$, as described in Eq. 5.

$$
\widehat{w}_{i}(t)=\widehat{t t}_{i}(t)-t t_{f_{-} i}
$$

Being a direct measurement, $\widehat{w}_{i}(t)$ is assumed as an accurate estimation of the current delay. This is compared to $w_{i}(t)$, obtained from the input - output diagram for the same period $\Delta T$, as described in the previous section, and which is flawed due to the detectors' count drift. The fusion algorithm consists in applying a correction factor, $\alpha_{i}(t)$, to the count at the upstream detector, $n_{u_{-} i}(t)$, in order to obtain a corrected virtual arrivals curve, $V_{i}^{*}(t)$, so that $\widehat{w}_{i}(t) \approx w_{i}(t)$. Considering $t_{c}$ as the current time instant where the travel time prediction is to be obtained, the data fusion correction algorithm is formulated in Eqs. 6 and 7, which can be solved using any numerical solver.

$$
\begin{gathered}
V_{i}^{*}(t)=V_{i}^{*}(t-\Delta t)+\alpha_{i}(t) n_{u i}(t) \quad \forall t \in\left(t_{c}-\Delta T, t_{c}\right) \\
\text { Find } \alpha_{i}(t) \text { so that } \widehat{w}_{i}(t) \approx w_{i}(t)
\end{gathered}
$$

Note that the correction is applied only to the upstream detector, without affecting the estimation of the outflow, $\bar{q}_{\text {out } i}^{(p)}$, which would affect the prediction. Also consider that it is advisable a frequent reset of the method, to prevent large drift errors leading to equally large correction factors. To that end, it is advisable to turn-off the algorithm when free-flowing traffic prevails. This is not restrictive in any sense, as in free-flow traffic any travel time estimation suffices (e.g. direct measurements or spot-speed methods from loop detectors; see (Soriguera and Robusté, 2011) for a detailed justification). Then, the data fusion algorithm and delay estimation from input-output diagrams should turn-on when some congestion is detected at current time $t_{c}$, and assuming free-flow at $t_{c}-\Delta T$ with null excess accumulation (i.e. $V_{i}(t)=D_{i}(t)$ in free-flowing at $t_{c}-\Delta T$ ). Congestion can be detected at $t_{c}$ by comparing the measured average speed (either at the upstream detector, downstream detector, or from GPS measurements if available) to a speed threshold, $v_{\text {ref }} i$, which should be calibrated for any specific application (e.g. usually a low percentile of the distribution of measured speeds during free-flowing periods; $v_{\text {ref_i }} \approx 80 \mathrm{~km} / \mathrm{h}$ can be generally accepted in multilane freeways).

For further reference about the fusion method, a similar procedure is proposed in (Soriguera and Martinez-Díaz, 2020) using ATT from AVI data instead of ITT. In this work, Bluetooth detectors were used as AVI devices.

\section{Case study and obtained results}

The proposed methodology has been tested with data obtained from simulation. $46 \mathrm{Km}$ of the 3-lane AP-7 freeway, near Barcelona (Spain) were simulated in the PTV-Vissim traffic microsimulator. Data for $3 \mathrm{~h}$ of heavy traffic with congestion were generated. From this simulation, a $12.8 \mathrm{Km}$ stretch of the freeway is chosen for the analysis. This is the same stretch used previously in (Soriguera and Martinez-Díaz, 2020). Two sections between loop detectors can be defined on the selected freeway stretch, one of them containing a junction (see Fig. 3).

The collected data from the simulation consisted in the vehicle counts, $n$, and time-mean speeds, $\bar{v}$, at all detector locations, as well as vehicle counts at the junction, all of them every $\Delta t$. For the construction of input - output diagrams, the net vehicle count at the junction is added to the closer detector (in this case the one at K.P. 113.9). This is necessary in order to keep the conservation of vehicles in the section and avoid the overcomplication of the construction of input - output diagrams. (Soriguera and Martinez-Díaz, 2020) justifies that the bias incurred by this procedure is small.

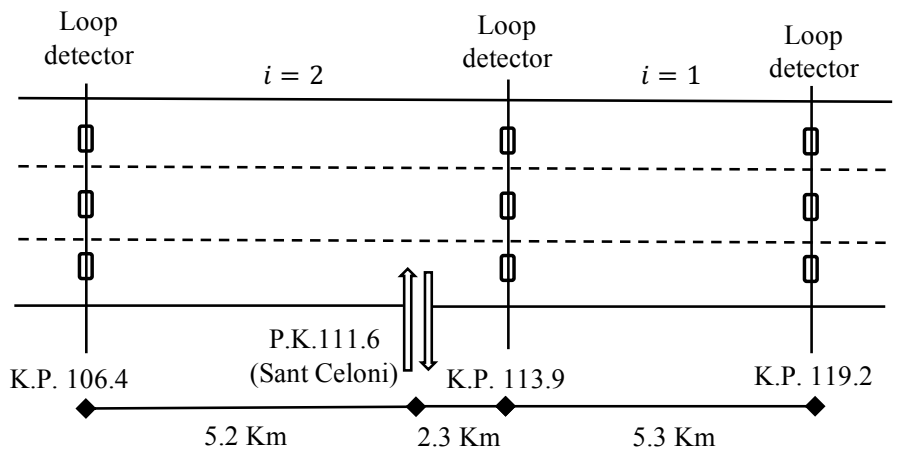

Fig. 3. Case study layout.

Additionally, the position, timestamp, and speed of a sample of $15 \%$ of the vehicles were registered with a frequency $\zeta$. These data are equivalent to that obtained either from GPS or mobile phone tracking. With these input data the method is of general application (i.e. it could be applied immediately without large investments), as loop detectors are still the most widespread source of traffic data, and $15 \%$ of tracked vehicles represents a low percentage which can be achieved with relative ease. Regarding $\zeta$, GPS chipsets allow up to $10 \mathrm{~Hz}$ signal updates, being $1 \mathrm{~Hz}$ the standard (i.e. 1 measurement per second; Martínez-Díaz, 2018). However, working with these high updating frequencies would involve huge storage and computing requirements at traffic management centers. Less demanding GPS signal frequencies are considered in the present case study. In addition, different values for $\Delta t$ and $\Delta T$, selected amongst those which are commonly used in practice, are considered in order to determine their influence on the final results. Table 1 shows the different combinations analyzed for $\zeta, \Delta t$ and $\Delta T$. Note that smaller frequencies for the GPS data measurements, $\zeta$, prevent using short ITT averaging periods, $\Delta T$, as the sample of GPS measurements would not be enough to compute a reliable ITT average.

Next, Table 2 presents the calibrated values for the other relevant variables of the method.

Table 1. Analyzed combinations of GPS data collection frequency and aggregation time intervals.

\begin{tabular}{ccc}
\hline GPS frequency $\zeta(\mathrm{Hz})$ & $\Delta t(\mathrm{~min})$ & $\Delta T(\mathrm{~min})$ \\
\hline \multirow{2}{*}{$1 / 12$} & 1 & 1 \\
& 1 & 3 \\
\multirow{2}{*}{$1 / 36$} & 3 & 3 \\
& 1 & 3 \\
\multirow{2}{*}{$1 / 60$} & 3 & 3 \\
& 1 & 3 \\
\end{tabular}

Table 2. Important parameters calibrated for the case study.

\begin{tabular}{lcc}
\hline \multicolumn{1}{c}{ Variable } & Units & Value \\
\hline Fraction of tracked vehicles & {$[\%]$} & 15 \\
Free-flow speed, $v_{f}$ & {$[\mathrm{Km} / \mathrm{h}]$} & 110 \\
Free-flow travel times, $t t_{f_{-} i}$ & {$[\mathrm{~min}]$} & $i=1 \rightarrow 2.89$ \\
Turn-on speed threshold, $v_{\text {ref }}$ & {$[\mathrm{Km} / \mathrm{h}]$} & 80 \\
$\begin{array}{l}\text { Duration of the moving average time } \\
\text { window to compute } \bar{q}_{\text {out }}^{(p)}, \tau\end{array}$ & {$[\mathrm{min}]$} & 15 \\
\hline
\end{tabular}


Table 3 and Figures 4 and 5 present the results obtained for the different scenarios analyzed. These figures show the travel times that would be disseminated by a real-time information system in the analyzed freeway stretch. The short-term predicted travel times from the proposed fusion algorithm (i.e. using inputoutput diagrams corrected with GPS data) are shown, together with the results of simply disseminating the ITT direct measurements from the GPS sampling, the common approach used in information systems based on directly measured travel times. Note that ITT measurements are updated every $\Delta T$, while new PTT estimations from input-output diagrams are reported every $\Delta t$ (despite drift correction factors, which depend on ITT measurements, are only updated every $\Delta T$ ). Table 3 shows the average absolute errors and the maximum errors for each scenario, with respect to the actually experienced travel times (i.e. defined as the "ground truth"). This ground truth travel time is computed, at the current time $t_{c}$, as the average travel time experienced by all vehicles entering the target section during the period $\left(t_{c}, t_{c}+\Delta t\right)$. Note that being future information, the real experienced travel times could not be known in real-time. However, in the present back-office analysis, "future" information is available for the whole period of analysis.

\begin{tabular}{|c|c|c|c|c|c|c|c|}
\hline $\begin{array}{c}\text { GPS } \\
\text { frequency } \\
\zeta(\mathrm{s})\end{array}$ & $\begin{array}{c}\Delta t \\
(\mathrm{~min})\end{array}$ & $\begin{array}{c}\Delta T \\
(\mathrm{~min})\end{array}$ & Method & $\begin{array}{c}\text { Mean absolute } \\
\text { error } \\
{[\mathrm{min}]}\end{array}$ & $\begin{array}{c}\text { Mean absolute } \\
\text { percentage } \\
\text { error [\%] }\end{array}$ & $\begin{array}{l}\text { Maximum } \\
\text { error } \\
\text { [min] }\end{array}$ & $\begin{array}{c}\text { Maximum } \\
\text { percentage error } \\
{[\%]}\end{array}$ \\
\hline \multirow[t]{6}{*}{12} & 1 & 1 & Directly disseminated ITT & 22.34 & 29.53 & -40.37 & -48.89 \\
\hline & & & Predicted travel times (data fusion algorithm) & 7.48 & 13.36 & -21.86 & -27.26 \\
\hline & 1 & 3 & Directly disseminated ITT & 21.69 & 28.60 & -40.14 & -48.62 \\
\hline & & & Predicted travel times (data fusion algorithm) & 12.24 & 18.56 & -28.24 & -35.21 \\
\hline & 3 & 3 & Directly disseminated ITT & 21.66 & 28.66 & -38.50 & -46.44 \\
\hline & & & Predicted travel times (data fusion algorithm) & 12.27 & 19.61 & -32.44 & -37.76 \\
\hline \multirow[t]{4}{*}{36} & 1 & 3 & Directly disseminated ITT & 18.12 & 31.05 & 48.46 & 91.83 \\
\hline & & & Predicted travel times (data fusion algorithm) & 12.23 & 18.50 & -28.14 & -35.09 \\
\hline & 3 & 3 & Directly disseminated ITT & 22.19 & 29.21 & -38.35 & -46.61 \\
\hline & & & Predicted travel times (data fusion algorithm) & 12.49 & 18.73 & -30.35 & -35.32 \\
\hline \multirow[t]{4}{*}{60} & 1 & 3 & Directly disseminated ITT & 19.06 & 29.59 & 48.32 & 91.57 \\
\hline & & & Predicted travel times (data fusion algorithm) & 8.77 & 15.05 & -21.88 & -28.57 \\
\hline & 3 & 3 & Directly disseminated ITT & 23.21 & 30.48 & -38.80 & -46.79 \\
\hline & & & Predicted travel times (data fusion algorithm) & 12.79 & 19.08 & -29.16 & -33.94 \\
\hline
\end{tabular}

Table 3. Comparison of travel time errors for different methods when disseminated in real-time.

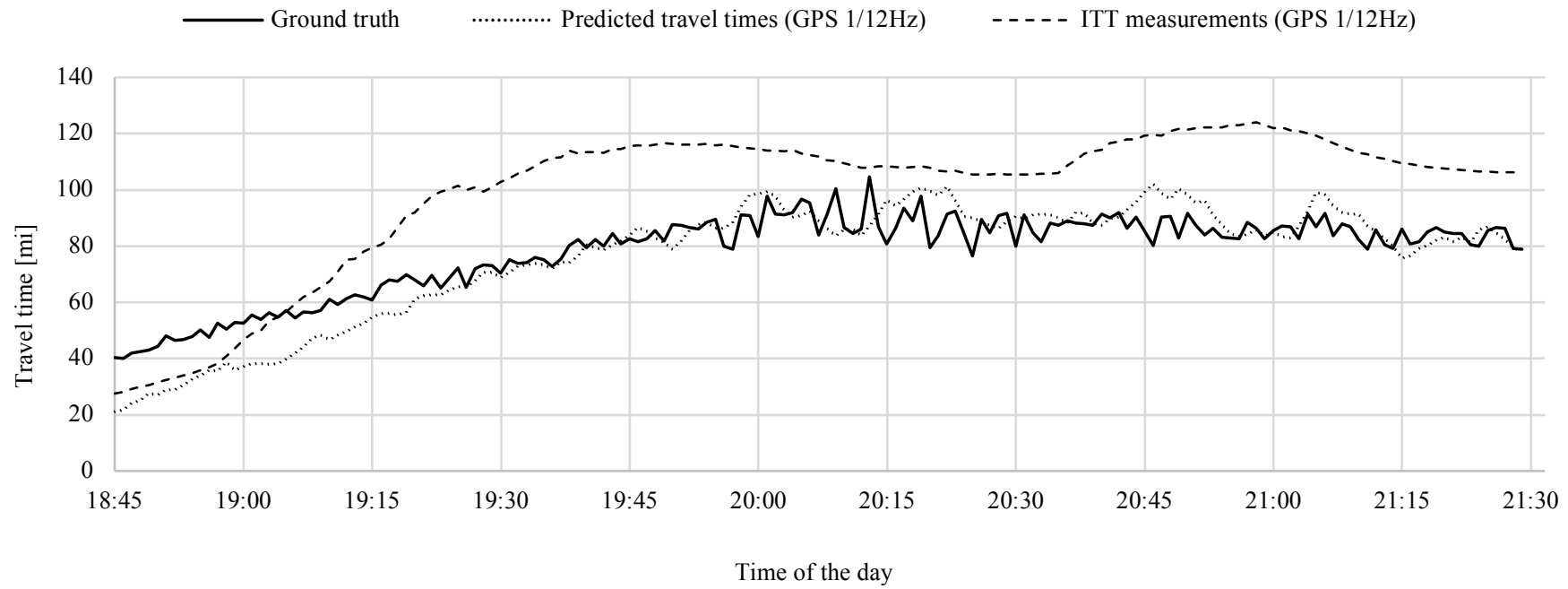

Fig. 4. Comparison of the suitability as real-time information of predicted travel times vs direct ITT measurements, with $\zeta=1 / 12 \mathrm{~Hz}, \Delta t=1 \mathrm{~min}$ and $\Delta T=1 \mathrm{~min}$. 


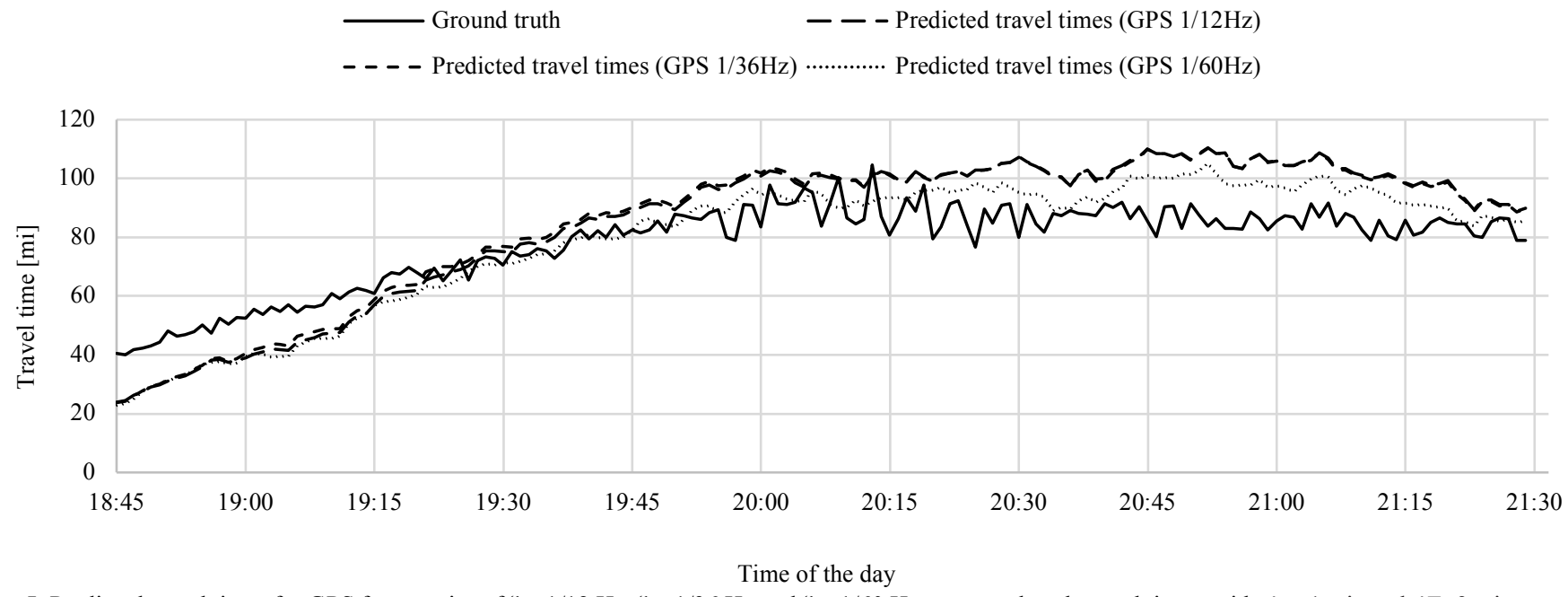

Fig. 5. Predicted travel times for GPS frequencies of $\zeta=1 / 12 \mathrm{~Hz}, \zeta=1 / 36 \mathrm{~Hz}$ and $\zeta=1 / 60 \mathrm{~Hz}$ vs ground truth travel times, with $\Delta t=1 \mathrm{~min}$ and $\Delta T=3$ min.

\section{Discussion}

In view of the results presented in Table 3 and Figure 4, it can be clearly seen that the application of the data fusion method is highly advantageous with respect to current practice of simply disseminating ITT measurements, in all the analyzed scenarios. For the best calibration of the method, the mean absolute percentage error is around 13\%, implying a significant performance improvement of the travel time information system. Maximum errors are also reduced reaching values around 30\% with respect to the ground truth, for the best calibration scenarios. Therefore, results show that the proposed methodology is very promising, as it improves the accuracy of predicted travel times while it could be applied immediately in most freeway contexts.

Further analysis of the obtained results allows analyzing the effects of the different parameters of the model. For instance, the frequency of GPS sampling $\zeta$, the fixed $15 \%$ tracked vehicles and the duration of the aggregation interval $\Delta T$, determine the number of GPS measurements used to compute the ITT. In addition, $\Delta T$ also affects the updating frequency of the drift correction. It can be seen in Table 3 that results are better for shorter $\Delta T$ (i.e. $\Delta T=1 \mathrm{~min}$ with respect to $\Delta T=3 \mathrm{~min}$ ). This means that a frequent update of the drift correction is preferable. However, this short $\Delta T$ requires higher frequency of the GPS measurements in order to obtain a large enough sample to compute a reliable ITT. Note that for $\Delta T=1 \mathrm{~min}$ a minimum of $\xi=1 / 12 \mathrm{~Hz}$ is required.

In contrast, if a larger $\Delta T$ is considered, the frequency of GPS sampling, $\zeta$, and the loop detector aggregation interval $\Delta t$ do not have a significant effect in the goodness of the results (see Table 3 and Fig. 5). This means that, if $\Delta T$ is large (e.g. $\geq 3 \mathrm{~min}$ ), low frequency GPS sampling provides enough measurements in order to estimate a reliable ITT capable of correcting the loop detectors drift. This could change in case there were much less vehicles in the target freeway stretch, but in this situation, freeflowing would prevail and the proposed method would not be necessary to obtain reliable travel time information. In turn, the accuracy of the input-output cumulative count method does not depend on $\Delta t$, although shorter $\Delta t$ 's allow a more frequent update of the travel time information.

Overall, these results prove that working with very high GPS frequencies is not necessary, as sometimes is postulated in the current era of big data. Traffic management centers do not need to worry much about their limited storage, communications and/or computing capacity, and most initiatives could be applied without huge infrastructure investments. This confirms similar findings in other prvious works (e.g. Sanaullah et al., 2016).

\section{Conclusions and further research}

Despite the coming revolution in vehicular traffic, travel time information systems will continue playing a key role in feeding traffic management strategies and providing valuable information to travelers. Efforts to improve these information systems by leveraging modern technologies, such as AVI or tracking systems, with smart data processing methods are being carried out. Although entailing a breakthrough with respect to traditional spot-speed methods based on loop detector measurements, the applicability of new methods and technologies as real-time information systems is limited. On the one hand, direct measurements of travel time are increasingly accurate but represent past information when used as real-time information systems. On the other hand, complex methodologies developed to provide travel time predictions generally require significant investment in technology and computational capacity, which cannot be achieved in a short period of time.

In this context, the present paper proposes a data fusion methodology that uses readily available technologies to provide short-term predictions of travel time, in order to feed real time information systems on freeways. Travel time predictions are obtained from the spatial information provided by input-output diagrams constructed from loop detector data. Cumulative count curves are used to compute the vehicular accumulation in the target freeway stretch from where to predict the time needed to serve the vehicles. The typical problem of the loop detector drift is corrected by means of a data fusion algorithm that takes advantage of the accuracy of a relatively small number of direct measurements, obtained from GPS tracking of a sample of vehicles. Today, most of the vehicles do travel with on-board GPS units, although only a fraction may transmit their information. The present application of the method assumes a conservative approach of $15 \%$ of vehicles tracked, and in a complicated scenario with very heavy congestion, the performance of the algorithm has proven to be very satisfactory. The goodness of the proposed methodology has been tested for different combinations of the calibration parameters, like the time intervals of data aggregation, the GPS sampling frequency or the updating interval for the travel time information. In all the scenarios the predictions of the proposed algorithm implied unquestionable improvements against the usual practice of simply disseminating direct measurements of travel time, reducing the MAPE approximately to half (i.e. from $30 \%$ to $15 \%)$. Furthermore, the present research work confirms that a high frequency of GPS sampling is not necessary to achieve good performance, a conclusion already obtained by previous works. Time intervals of around 1 minute between GPS measurements of the tracked vehicles might suffice, which helps in reducing 
costs and computational efforts.

Regarding the direct applicability of the method with the data readily available at traffic management centers, probably the requirement of GPS tracking data as an input to correct the loopdetector drift, is the most challenging need. To that end, it should be recognized that GPS on board devices travel in almost all vehicles (e.g. in form of smartphones), and traffic information and routing apps are increasingly popular, meaning more vehicles sharing their GPS tracking data. In this context, it is not adventurous to consider that tracking data from $15 \%$ of vehicles are available. It is true, however, that these data are collected by ICT companies, and not directly by traffic agencies, as traditionally done. This means that cooperation between the traffic administration and ICT companies will be needed to exploit the benefits of technology. This need, which will go beyond the field of traffic information, will be surely fulfilled for the sake of the progress.

Further research should address a deeper sensitivity analysis of this type of methodologies, to derive more recommendations for its transferability. Factors such as the fraction of tracked vehicles, the level of congestion for which these systems pay-off, the optimal length of the target sections, or the treatment of on/off-ramps and their relative flows, will need to be assessed. In addition, the method is sensitive to the failure of loop-detectors, and therefore it would be advisable the integration with an algorithm to account for possible loop detector data losses. In the medium-long term, further integration with artificial intelligence in order to deal with the challenges of traffic management in the era of autonomous driving is another ambitious purpose to pursue. Being simple in the conceptual foundations and requiring a small amount of data, will make the proposed method an excellent complement and safeguard to more sophisticated systems with much more challenging applicability requirements.

\section{Acknowledgments}

This research has been partially funded by the Spanish Ministry of Science and Innovation (Ministerio de Ciencia e Innovación, Gobierno de España), within the Program for Research Aimed at the Society's Challenges (grant ref. PID2019105331RB-I00). Authors especially acknowledge the work of Enrique Jiménez, who provided us with the simulation data to perform the case study.

\section{Disclosure statement}

This research is not affected by any conflict of interest.

\section{References}

Abbott-jard, M., H. Shah, and A. Bhaskar. (2013). Empirical evaluation of Bluetooth and Wifi scanning for road transport. 36th Australasian Transport Research Forum (ATRF), October 2nd-4th, Brisbane.

Barceló, J. and M. Kuwahara. (2010). Traffic data collection and its standardization. Springer, Berlin.

Bar-Gera, H. (2007). Evaluation of a cellular phone-based system for measurements of traffic speeds and travel times: A case study from Israel. Transportation Research Part C: Emerging Technologies 15(6), 380-391.

Ben-Akiva, M., M. Bierlaire, D. Burton, H.N. Koutsopoulos and R. Mishalani. (2002). Network state estimation and prediction for real-time transportation management applications. Transportation Research Board Annual Meeting, CD-ROM. National Academies Press, Washington, DC, USA.

Bhaskar, A. and E. Chung. (2013). Fundamental understanding on the use of Bluetooth scanner as a complementary transport data. Transportation Research Part C: Emerging Technologies 37, 42-72.

Buisson, C. (2006). Simple traffic model for a simple problem: sizing travel time measurement devices. Transportation Research Record: Journal of the Transportation Research Board 1965, 210-218.

Cedillo-Campos, M.G., C.M. Pérez-González, J. Piña-Barcena and E. Moreno-Quintero. (2019). Measurement of travel time reliability of road transportation using GPS data: A freight fluidity approach. Transportation Research Part A: Policy and Practice 130, 240-288.
Chen, C., X. Zhao, Y. Zhang, J. Rong and X. Liu. (2019). A graphical modeling method for individual driving behavior and its application in driving safety analysis using GPS data. Transportation Research Part F: Traffic Psychology and Behaviour 63, 118-134.

Chien, S.I.J. and C.M. Kuchipudi. (2003). Dynamic travel time prediction with real-time and historic data. Journal of Transportation Engineering 129 (6), 608-616.

Chu, L.Y., J.S. Oh and W. Recker. (2005). Adaptive Kalman filter based freeway travel time estimation. Transportation Research Board 84th Annual Meeting CD-ROM. National Academies Press, Washington, DC, USA.

Coifman, B. and S. Krishnamurthya. (2007). Vehicle reidentification and travel time measurement across freeway junctions using the existing detector infrastructure. Transportation Research Part C: Emerging Technologies 15(3), 135-153.

Daganzo, C.F. (1983). Derivation of delays based on input-output analysis. Transportation Research Part A 17(5), 341-342.

Daganzo, C.F. (2007). Urban gridlock: Macroscopic modeling and mitigation approaches. Transportation Research Part B: Methodological 41(1), 4962.

Edie, L.C. (1965). Discussion of traffic stream measurements and definitions. Proc. 2nd International Symposium on the Theory of Traffic Flow, J. Almond, ed., OECD, Paris, 139-154.

Ge, Q. and D. Fukuda. (2016). Updating origin-destination matrices with aggregated data of GPS traces. Transportation Research Part C: Emerging Technologies 69, 291-312.

Ge, J.I, S.S. Avedisov, C. R. He, W. B. Qin, M. Sadeghpour and G. Orosz. (2018). Experimental validation of connected automated vehicle design among human-driven vehicles. Transportation Research Part C: Emerging Technologies 91, 335-352.

Herrera, J. C., D. Work, X. Ban, R. Herring, Q. Jacobson and A. Bayen. (2010). Evaluation of traffic data obtained via GPS-enabled mobile phones: the Mobile Century field experiment. Transportation Research Part C: Emerging Technologies 18, 568-583.

$\mathrm{Hu}$, T.Y. (2001). Evaluation framework for dynamic vehicle routing strategies under real-time information. Transportation Research Record 1774, 115-122.

Ishak, S. and H.M. Al-Deek. (2002). Performance evaluation of short-term time-series traffic prediction model. Journal of Transportation Engineering 128 (6), 490-498.

Kemajou, A., R. Jaligot, M. Bosch and J. Chenal. (2019). Assessing motorcycle taxi activity in Cameroon using GPS devices. Journal of Transport Geography 79, 102472.

Knapen, L., T. Bellemans, D. Janssens and G. Wets. (2018). Likelihoodbased offline map matching of GPS recordings using global trace information. Transportation Research Part C: Emerging Technologies 93, $13-35$.

Krause, C.M. and L. Zhang. (2019). Short-term travel behavior prediction with GPS, land use, and point of interest data. Transportation Research Part B: Methodological 123, 349-361.

Laha, A.K. and S. Putatunda. (2018). Real time location prediction with taxiGPS data streams. Transportation Research Part C: Emerging Technologies 92, 298-322.

Lam, S.H. and T.D. Toan .(2008). Short-term travel time predictions using support vector regression. Transportation Research Board Annual Meeting, CD-ROM. National Academies Press, Washington, DC, USA.

Laoide-Kemp, D., and M. O'Mahony. (2020). Dealing with latency effects in travel time prediction on motorways. Transportation Engineering 2, 100009 .

Leduc, G. (2008). Road traffic data: collection methods and applications. Working Papers of the JRC on Energy, Transport and Climate Change 1, European Commission.

Martínez-Díaz, M. (2018). Highway travel time information systems: from traditional to cooperative driving environments. ( $\mathrm{PhD}$ Dissertation). Universidade da Coruña.

Min, W. and L. Wynter. (2011). Real-time road traffic prediction with spatiotemporal correlations. Transportation Research Part C 19 (4), 606-616.

Mori, U., A. Mendiburu, M. Álvarez and J.A. Lozano. (2015). A review of travel time estimation and forecasting for Advanced Traveller Information Systems. Transportmetrica A: Transport Science 11(2), 119-157.

Nanthawichit, C., T. Nakatsuji and H. Suzuki. (2003). Application of probevehicle data for real-time traffic-state estimation and short-term trave 1timeprediction on a freeway. Transportation Research Record 2987, 4959.

Newell, G. F. (1993). A simplified theory of kinematic waves in highway traffic, I general theory, II queuing at freeway bottlenecks, III multidestination flows. Transportation Research Part B: Methodological 27(1), 281-313.

Okutani, I. and Y.J. Stephanedes. (1984). Dynamic prediction of traffic volume through Kalman filtering theory. Transportation Research Part B 18 (1), 1-11.

Park, D. and L. Rilett. (1999). Forecasting freeway link travel times with a multilayer feed forward neural network. Computer-Aided Civil and Infrastructure Engineering 14 (5), 357-367. 
Patire, A.D., M. Wright, B. Prodhomme and A.M. Bayen. (2015). How much GPS data do we need?. Transportation Research Part C: Emerging Technologies 58(B), 325-342.

Rilett, L.R. and D. Park. (2001). Direct forecasting of freeway corridor travel times using spectral basis neural networks. Transportation Research Record 1752, 140-147.

Sanaullah, I., M. Quddus and M. Enoch. (2016). Developing travel time estimation methods using sparse GPS data. Journal of Intelligent Transportation Systems 20(6), 532-544.

Simoncini, M., L. Taccari, F. Sambo, S. Salti, A. Lori and L. Bravi. (2018). Vehicle classification from low-frequency GPS data with recurrent neural networks. Transportation Research Part C: Emerging Technologies 91, 176-191.

Smulders, S.A., A. Messmer and W.J.J. Knibbe. (1999). Real-time application of METANET in traffic management centres. In: Proceedings of the 6th World Congress on Intelligent Transport Systems (ITS), Toronto, Canada.

Soriguera, F., D. Rosas and F. Robusté. (2010). Travel time measurement in closed toll highways. Transportation Research Part B 44 (10), 1242-1267.

Soriguera, F. and F. Robusté. (2011). Requiem for freeway travel time estimation methods based on blind speed interpolations between point measurements. IEEE Transactions on Intelligent Transportation Systems 12 (1), 291-297.

Soriguera, F. and F. Robusté. (2011). Highway travel time accurate measurement and short-term prediction using multiple data sources. Transportmetrica 7(1), 85-109.

Soriguera, F. (2014). On the value of highway travel time information systems. Transportation Research Part A: Policy and Practice 70, 294310 .

Soriguera, F. and M. Martínez-Díaz (2020). Freeway travel time information from input-output vehicle counts: A drift correction method based on AVI data. IEEE Transactions on Intelligent Transportation Systems, In press.

Sun, H., H.X. Liu, H. Xiao, R.R. He and B. (2003). Short-term traffic forecasting using the local linear regression model. Transportation Research Board Annual Meeting CD-ROM. National Academies Press, Washington, DC, USA.

Turner, S. M., W.L. Eisele, R.J. Benz and D.J. Holdener. (1998). Travel time data collection handbook. Research Report FHWA-PL-98-035. Washington, D.C. Federal Highway Administration.

Unde, M. D. and B. Borkar. (2014). Remote vehicle tracking and driver health monitoring system using GSM modem and Google maps. (IJCSIT) International Journal of Computer Science and Information Technologies 5(3), 2828-2832.

van Hinsbergen, C.P., J.W.C. van Lint and F.M. Sanders. (2007). Short term traffic prediction models. Proceedings of the 14th World Congress on Intelligent Transport Systems (ITS), 1-18, Beijing.

van Hinsbergen, C.P., J.W.C. van Lint and H.J. van Zuylen. (2009). Bayesian committee of neural networks to predict travel times with confidence intervals. Transportation Research Part C 17 (5), 498-509.

van Lint, J.W.C., S.P. Hoogendoorn and H.J. Van Zuylen. (2002). Freeway travel time prediction with state-space neural networks - modeling statespace dynamics with recurrent neural networks. Transportation Research Record 1811, 30-39.

van Lint, J.W.C. and N.J. Van der Zijpp. (2003). Improving a travel time estimation algorithm by using dual loop detectors. Transportation Research Record 1855, 41-48.

van Lint, J.W.C. and N.J. Van der Zijpp. (2003). Improving a travel time estimation algorithm by using dual loop detectors. Transportation Research Record 1855, 41-48.

van Lint, J.W.C., S.P. Hoogendoorn and H.J. van Zuylen. (2005). Accurate freeway travel time prediction with state-space neural networks under missing data. Transportation Research Part C 13 (5-6), 347-369.

van Lint, J.W.C. (2006). A reliable real-time framework for short-term freeway travel time prediction. Journal of Transportation Engineering 132 (12), 921-932.

van Lint, J.W.C. (2008). Online learning solutions for freeway travel time prediction. IEEE Transactions on Intelligent Transportation Systems 9 (1), 38-47.

Vlahogianni, E.I. and E.N. Barmpounakis. (2017). Driving analytics using smartphones: algorithms, comparisons and challenges. Transportation Reseach Part C: Emerging Technologies 79, 196-206.

Wang, Y., L. Wei and P. Chen. (2020). Trajectory reconstruction for freeway traffic mixed with human-driven vehicles and connected and automated vehicles. Transportation Research Part C: Emerging Technologies 11, 135-155.

Wei, C. and Y. Lee. (2007). Development of freeway travel time forecasting models by integrating different sources of traffic data. IEEE Transactions on Vehicular Technology 56 (6), 3682-3694.

Xia, J., M. Chen, and W. Huang. (2011). A multistep corridor travel-time prediction method using presence-type vehicle detector data. Journal of Intelligent Transportation Systems 15 (2), 104-113.

Yang, J.S. (2005). A study of travel time modeling via time series analysis. In: Proceedings of IEEE Conference on Control Applications, Toronto, Canada. 855-860.
Zeng, X. and Y. Zhang, Y. (2013). Development of recurrent neural network considering temporal-spatial input dynamics for freeway travel time modeling. Computer-Aided Civil and Infrastructure Engineering 28, 359 371.

Zhang, X. and J.A. Rice. (2003). Short-term travel time prediction. Transportation Research Part C: Emerging Technologies 11 (3-4), $187-$ 210.

Zhang, Y. and A. Haghani. (2015). A gradient boosting method to improve travel time prediction. Transportation Research Part C: Emerging Technologies 58, 308-324.

Zhu, J. and X. Ye. (2018). Development of destination choice model with pairwise district-level constants using taxi GPS data. Transportation Research Part C: Emerging Technologies 93, 410-424. 